\title{
The Role of Anesthesiology in Global Health: A Comprehensive Guide
}

\author{
Ram Roth, Clifford Gevirtz, Elizabeth A. M. Frost, Carrie L. H. Atcheson. Springer \\ International Publishing, Switzerland 2015, 432 pages. ISBN 978-3-319-09422-9
}

\author{
Gregory Klar, MD
}

Received: 27 February 2015/Accepted: 9 April 2015/Published online: 21 April 2015

(C) Canadian Anesthesiologists' Society 2015

The global burden of surgical disease is an important cause of death and disability in low- and middle-income countries (LMICs); however, surgical and anesthesia services have been traditionally neglected in LMICs. There is a critical shortage of trained anesthesia providers in LMICs who can respond to a large and growing surgical load. Furthermore, the lack of trained anesthesia providers contributes to the high perioperative mortality. Anesthesia is becoming an increasingly important player in international health with growing numbers of anesthesiologists working abroad. The recently published book, The Role of Anesthesiology in Global Health: A Comprehensive Guide, reviews international health literature pertinent to anesthesiologists and serves as a guide for the anesthesiologist considering or already involved in international work.

The book is subdivided into three large sections. Part I, "Evolution and Development of Missions", comprises a description of the history of foreign aid, a review of the literature on the global burden of surgical disease, and the essentials to prepare anesthesiologists for work in a lowresource setting. Dr. Kelly McQueen eloquently depicts the critical shortage of anesthesia providers in light of the growing surgical burden (Chapter 3), and Dr. Enright highlights key aspects of work in a resource-limited setting (Chapter 6). Part II, "Specific Case-Related Implementation", emphasizes lessons learned from experience and the "real-life" difficulties of work in a lowresource setting. A range of topics are considered, including subspecialty anesthesia practice and perioperative care in the

G. Klar, MD ( $\square)$

Department of Anesthesia, University of Manitoba, Winnipeg,

MB, Canada

e-mail: gklar@rcsi.ie war-disaster setting. Part III, "Education at Home and Abroad", speaks to the importance of harmonizing international health efforts to meet the needs of LMICs. This section discusses the importance of sustainable education for both the receiving medical institutions as well as the international health workers. The role of education for physicians in training and medical students who are interested in global health is also highlighted.

This informative well-written textbook is one of the first books to discuss anesthesia in the context of global health. Its publication is a timely response to the growing interest in anesthesia care in LMICs among anesthesiologists and anesthesia trainees. Authors participating in international health delivery impart their global experience and knowledge in this multi-authored text. The text is an engaging read offering anecdotes of first-hand experience balanced with evidence from the international health literature. This book is perfectly suited to anesthesiologists interested in global health, yet it offers a wealth of information for all anesthesia providers. Furthermore, this textbook provides a solid reference for other physicians, particularly surgeons interested in work in international health. This publication could be used to complement and support existing modules and workshops in anesthesia international health education.

In summary, I view this work as a novel and important anesthesia textbook. The overall tone and message resonate with other international health literature which stresses the importance of harmonization, development, and sustainability. In my view, this book combines a wealth of personal experience with the best available literature to prepare anesthesiologists for work in the global health sector.

Conflicts of interest None declared. 